\title{
Mite is Right: A Case of Infected Scabies Masquerading as a Blistering Disorder
}

\author{
Ahsan Tameez-ud-din ${ }^{1}$, Asim Tameez ud din², and Ifrah Malik ${ }^{1}$ \\ ${ }^{1}$ Rawalpindi Medical University \\ ${ }^{2}$ Shifa International Hospitals Ltd
}

June 30, 2021

\begin{abstract}
Scabies may present with unusual features like vesicles, pustules, and bullae. In the setting of an already burdened health sector, careful history taking is of utmost importance in order to avoid unnecessary and expensive investigations to reach a diagnosis.
\end{abstract}

Mite is Right: A Case of Infected Scabies Masquerading as a Blistering Disorder Authors:

Ahsan Tameez-ud-din MBBS ${ }^{1 *}$, Asim Tameez Ud Din MD, MBBS ${ }^{2}$, Ifrah Malik MBBS ${ }^{3}$

1. House Physician, Dermatology Department, Benazir Bhutto Hospital, Rawalpindi, Pakistan

2. Internal Medicine Resident, Shifa International Hospital, Islamabad, Pakistan

3. House Physician, Psychiatry Department, Benazir Bhutto Hospital, Rawalpindi, Pakistan

*Corresponding Author: Ahsan Tameez-ud-din

Email Address: ahsantameezuddinmalik@gmail.com

Contact number: +923315166726

KEY WORDS : scabies, blisters, erosions

\begin{abstract}
Scabies may present with unusual features like vesicles, pustules, and bullae. In the setting of an already burdened health sector, careful history taking is of utmost importance in order to avoid unnecessary and expensive investigations to reach a diagnosis.
\end{abstract}

\section{Key Clinical Message}

Scabies classically presents with itching and skin lesions in the interdigital spaces of hands, trunk, and genitals. Rarely, non-classical skin lesions can result in misdiagnosis and inappropriate management of patients.

\section{Case Presentation}

A 15-year-old boy presented with a history of flaccid blister eruption on all four limbs and genital area for 2 months. It was preceded by intense pruritis leading to the appearance of fluid filled vesicles and pustules (Figure 1). They ruptured within a day, leading to skin erosions with crusting in some areas (Figures 2,3). There was no family history of autoimmune disorders. 
The patient was admitted with differential diagnoses of Linear IgA disease and Chronic bullous disease of childhood (CBDC). His blood tests revealed raised Total Leukocyte Count and he was started on culture based oral antibiotics. Inconclusive skin biopsy report, unsatisfactory response to steroids in past and clues from family history regarding presence of pruritic, papular lesions in 2 siblings pointed towards infected scabies as a probable diagnosis. Initiating oral Ivermectin resulted in a drastic improvement in the lesions and intensity of pruritis over the next couple of days. He was discharged with rigorous counselling of patient and family regarding the correct application of topical scabicide (Permethrin 5\% applied on day 1 and 7 ) and follow-up after 2 weeks revealed that the lesions and the symptoms had resolved completely.

Scabies classically presents with pruritus and skin lesions (papules, nodules, burrows) in the interdigital spaces of hands, trunk and genitals [1]. Atypical skin lesions like vesicles, pustules and bullae or superimposed infection of skin may result in misdiagnosis and inappropriate patient management [2]. In such cases, detailed history of the patient and acquaintances should be sought.

\section{Data Availability Statement}

The data that support the findings of this study are available from the corresponding author upon reasonable request.

\section{Legends}

Figure 1: Arrow shows flaccid blisters around the ankle

Figure 2: Arrow shows crusted lesions and erosions

Figure 3: Shows crusted lesions (red arrow) and erosions (yellow arrow)

\section{Author Contributions}

Ahsan Tameez-ud-din was involved in patient care, provided necessary data for the article and suggested the title. Asim Tameez-ud-din was involved in manuscript preparation. Ifrah Malik checked the manuscript for grammatical and scientific errors.

\section{REFERENCES}

Chandler DJ, Fuller LC. A review of scabies: an infestation more than skin deep. Dermatology. 2019;235(2):79-90.

Gutte R. Bullous scabies in an adult: A case report with review of literature. Indian Dermatology Online Journal. 2013 Oct;4(4):311. 

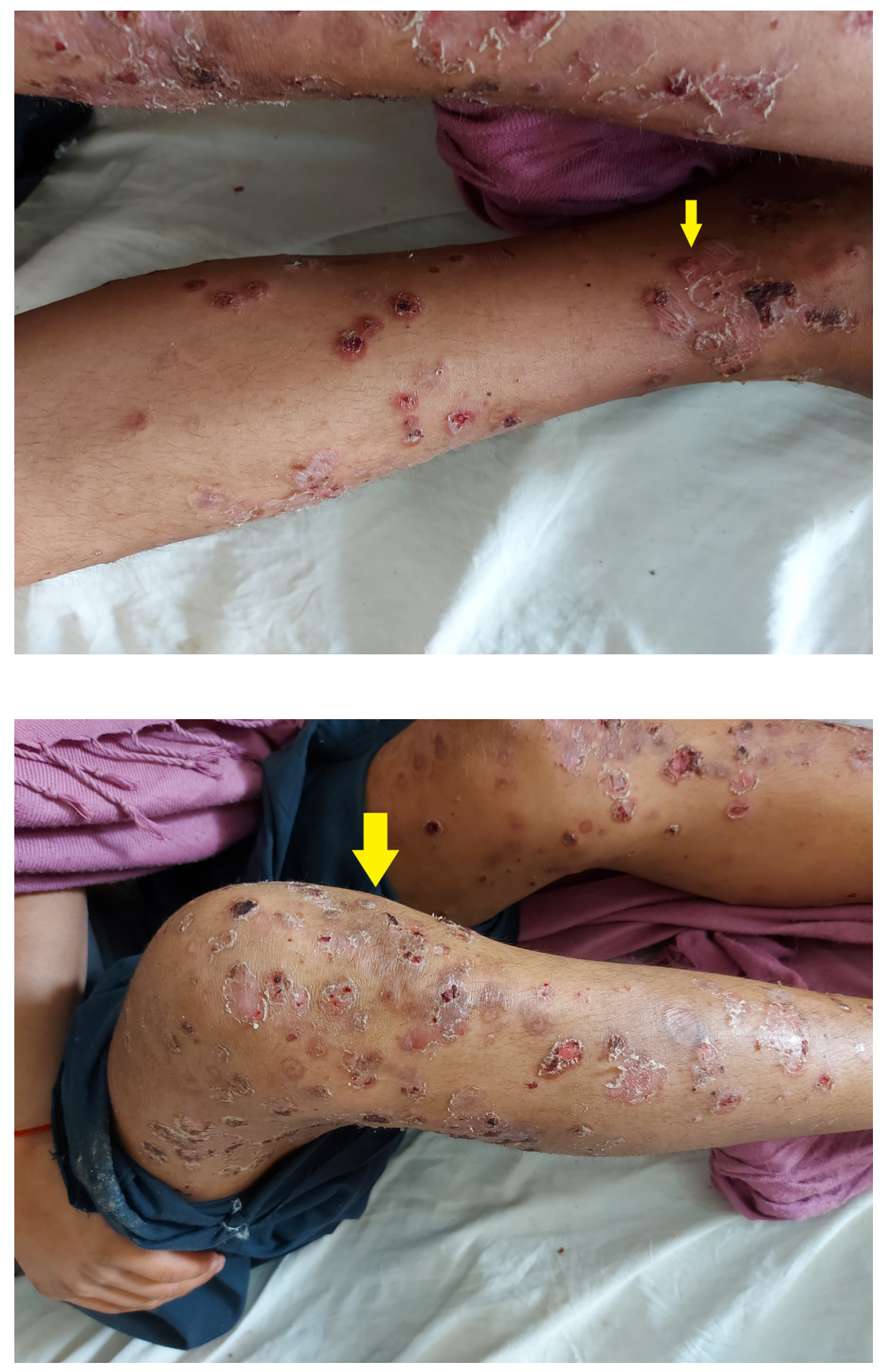


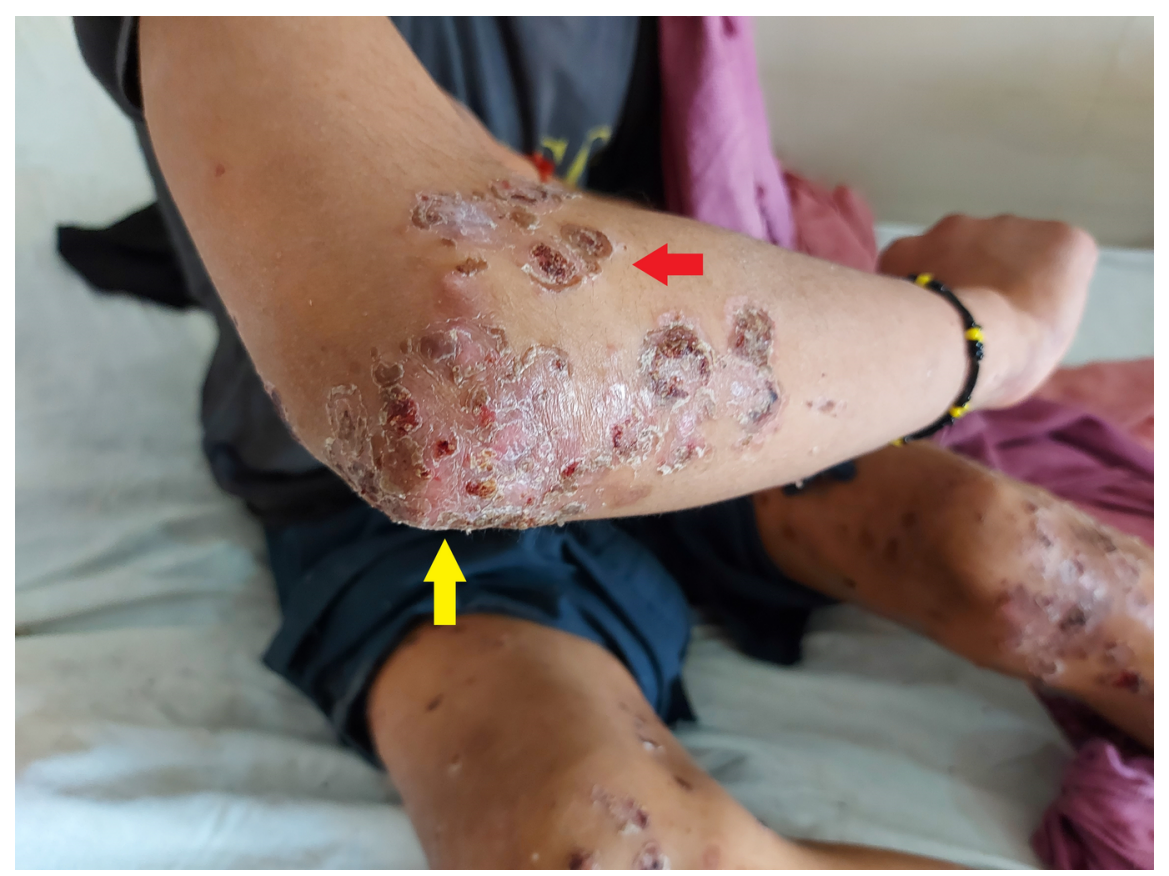

